\title{
A NOTE ON COMPACTLY GENERATED CO-t-STRUCTURES
}

\author{
DAVID PAUKSZTELLO
}

\begin{abstract}
The idea of a co-t-structure is almost 'dual' to that of a $t$ structure, but with some important differences. This note establishes co$t$-structure analogues of Beligiannis and Reiten's corresponding results on compactly generated $t$-structures.
\end{abstract}

The notion of a co-t-structure on a triangulated category was introduced independently by the author in [15] and Bondarko in [7]. In [7] they are referred to as weight structures; in this note we continue the terminology of [15]. In [7] they are introduced in the context of understanding Grothendieck's weight filtration in Voevodsky's triangulated category of motives; see also [6]. In [1] co- $t$-structures are important ingredients in the proofs of purity and decomposition theorems for 'staggered sheaves', and in [18] and [19] they are studied in the context of Chow motives and Artin-Tate motives, respectively. In the representation theoretic setting, co-t-structures have recently been studied in connection with the Auslander-Buchweitz context in [13].

In view of their recent proliferation into different branches of mathematics, it is useful to establish basic results regarding the structure and behaviour of co-t-structures, and, in particular, their relation with, similarities to, and differences from $t$-structures. In this sense, the present note should be viewed as an extension of [15] providing co- $t$-structure analogues of the corresponding results for $t$-structures in [4].

Throughout this note, $\mathscr{T}$ will be a triangulated category with set indexed coproducts and and $\Sigma: \mathscr{T} \rightarrow \mathscr{T}$ will denote its suspension functor. We direct the reader to [9] for an introduction to triangulated categories. We first recall some definitions. An object $S$ of $\mathscr{T}$ is called rigid if $\operatorname{Hom}_{\mathscr{T}}\left(S, \Sigma^{i} S\right)=0$ for all $i>0$ (see [11]); $S$ is called compact if for any set indexed family of objects $\left\{X_{i}\right\}_{i \in I}$ of $\mathscr{T}$ one has the natural isomorphism $\operatorname{Hom}_{\mathscr{T}}\left(S, \coprod_{i \in I} X_{i}\right) \cong$ $\coprod_{i \in I} \operatorname{Hom}_{\mathscr{T}}\left(S, X_{i}\right)$. Recall also that a generating set for $\mathscr{T}$ is a set of objects $\mathscr{G}$ such that if $\operatorname{Hom}_{\mathscr{T}}(G, X)=0$ for all $G \in \mathscr{G}$ then $X=0$.

In [10, Theorem 1.3], if one takes a compact rigid object $S$ of $\mathscr{T}$ such that $\left\{\Sigma^{i} S \mid i \in \mathbb{Z}\right\}$ is a generating set, then one obtains a canonical $t$-structure on $\mathscr{T}$

Date: 12 th October 2010.

2000 Mathematics Subject Classification. 18E30, 18E40.

Key words and phrases. Triangulated category, compact object, co-t-structure. 
given by

$$
\begin{aligned}
\mathscr{X} & =\left\{X \in \mathscr{T} \mid \operatorname{Hom}_{\mathscr{T}}\left(S, \Sigma^{i} X\right)=0 \text { for } i>0\right\}, \\
\mathscr{Y} & =\left\{X \in \mathscr{T} \mid \operatorname{Hom}_{\mathscr{T}}\left(S, \Sigma^{i} X\right)=0 \text { for } i<0\right\} .
\end{aligned}
$$

In [4, Theorem III.2.3], it is established that any compact object $S$ of $\mathscr{T}$ induces a canonical $t$-structure on $\mathscr{T}$ given by

$$
\begin{aligned}
\mathscr{X} & =\Sigma^{-1}\left({ }^{\perp} \mathscr{Y}\right), \\
\mathscr{Y} & =\left\{X \in \mathscr{T} \mid \operatorname{Hom}_{\mathscr{T}}\left(S, \Sigma^{i} X\right)=0 \text { for } i<0\right\} .
\end{aligned}
$$

Since the 'torsion-free class' $\mathscr{Y}$ of a $t$-structure is always closed under nonpositive suspensions, this description of $\mathscr{Y}$ is natural. However, an obvious question is whether the 'torsion class' $\mathscr{X}$ also has such a nice description. In [4, Proposition III.2.8], this is shown to be the case if and only if the hypotheses of [10, Theorem 1.3] hold. A natural question is thus: what happens if one specifies the 'torsion class' $\mathscr{X}=\left\{X \in \mathscr{T} \mid \operatorname{Hom}_{\mathscr{T}}\left(S, \Sigma^{i} X\right)=0\right.$ for $\left.i>0\right\}$ and sets $\mathscr{Y}=\Sigma\left(\mathscr{X}^{\perp}\right)$ ? Unfortunately, it seems that this is not possible in general for $t$-structures but the situation arises naturally in the setting of co- $t$ structures.

Another motivation is that the theory of co-t-structures seems to be richer when there exist adjacent $t$-structures (see [7, Sections 4.4 and 4.5]). However, as remarked in [7, Remark 4.5.3], the question of existence of an adjacent co- $t$ structure when a triangulated category is endowed with a $t$-structure is difficult in general. The main result of this note provides a case where such adjacent (co)-t-structures exist.

We now recall the definition of a co-t-structure:

Definition 1 ([15], Definition 2.4). Let $\mathscr{T}$ be a triangulated category with set indexed coproducts. A pair of full subcategories of $\mathscr{T},(\mathscr{A}, \mathscr{B})$, is called a co-t-structure on $\mathscr{T}$ if it satisfies the following properties:

(0) $\mathscr{A}$ and $\mathscr{B}$ are closed under direct summands;

(1) $\Sigma^{-1} \mathscr{A} \subseteq \mathscr{A}$ and $\Sigma \mathscr{B} \subseteq \mathscr{B}$;

(2) $\operatorname{Hom}_{\mathscr{T}}\left(\Sigma^{-1} \mathscr{A}, \mathscr{B}\right)=0$;

(3) For any object $X$ of $\mathscr{T}$ there exists a distinguished triangle $\Sigma^{-1} A \rightarrow$ $X \rightarrow B \rightarrow A$ with $A \in \mathscr{A}$ and $B \in \mathscr{B}$.

In [15, Definition 3.2] an object $S$ of $\mathscr{T}$ is called a simply connected corigid object of $\mathscr{T}$ if

(1) $S$ is corigid, that is, $\operatorname{Hom}\left(\Sigma^{i} S, S\right)=0$ for $i>0$;

(2) $\operatorname{Hom}_{\mathscr{T}}(S, \Sigma S)=0$;

(3) $\operatorname{End}(S)$ is a division ring.

We shall refer to $S$ as a connected corigid object of $\mathscr{T}$ if the first two conditions hold. 
Motivated by the observation that the case studied in [4] and [10] represents a 'chain situation', and that, often, a 'cochain situation' is more natural (see [15] for more remarks on this), the author showed in [15, Theorem 3.2] that if $S$ is a simply connected corigid object of $\mathscr{T}$ and $\left\{\Sigma^{i} S \mid i \in \mathbb{Z}\right\}$ is a generating set for $\mathscr{T}$, then one has a canonical co-t-structure on $\mathscr{T}$ given by

$$
\begin{aligned}
\mathscr{A} & =\left\{X \in \mathscr{T} \mid \operatorname{Hom}_{\mathscr{T}}\left(S, \Sigma^{i} X\right)=0 \text { for } i<0\right\}, \\
\mathscr{B} & =\left\{X \in \mathscr{T} \mid \operatorname{Hom}_{\mathscr{T}}\left(S, \Sigma^{i} X\right)=0 \text { for } i>0\right\} .
\end{aligned}
$$

Observe that the class of objects $\mathscr{B}$ above coincides with the 'torsion class' $\mathscr{X}$ of (1). It is natural to ask, therefore, whether analogous theorems to those in [4] hold in the case of co-t-structures. This is indeed the case. In this note, we prove the corresponding results for co-t-structures and observe that in the first part of [15] the hypotheses can be relaxed so that $S$ is a connected corigid object (i.e. $\operatorname{End}(S)$ is a division ring is not required) and are, in addition, necessary and sufficient.

Recall from [15] that a co-t-structure on a triangulated category $\mathscr{T}$ is called non-degenerate if $\cap_{n \in \mathbb{Z}} \sum^{n} \mathscr{A}=\cap_{n \in \mathbb{Z}} \sum^{n} \mathscr{B}=0$; following [4] we shall say it is of finite type if $\mathscr{B}$ is closed under set indexed coproducts. Consider the following setup.

Setup 2. Let $\mathscr{T}$ be a triangulated category with set indexed coproducts. Suppose $\mathscr{S}$ is a set of compact objects in $\mathscr{T}$. Let $\mathscr{R}=\left\{\Sigma^{i} S \mid S \in \mathscr{S}, i<0\right\}$. Define the following full subcategories of $\mathscr{T}$ :

$$
\begin{aligned}
\mathscr{A} & :=\Sigma\left({ }^{\perp} \mathscr{B}\right) \\
\mathscr{B} & :=\left\{X \in \mathscr{T} \mid \operatorname{Hom}_{\mathscr{T}}\left(S, \Sigma^{n} X\right)=0 \text { for all } S \in \mathscr{S} \text { and } n>0\right\} .
\end{aligned}
$$

Note that $\mathscr{B}=\mathscr{R}^{\perp}$ and $\operatorname{Add}(\mathscr{R})^{\perp}=\mathscr{R}^{\perp}$, where $\operatorname{Add}(\mathscr{R})$ denotes the smallest full subcategory closed under direct summands of arbitary coproducts of objects of $\mathscr{R}$.

Recall that an additive category $\mathscr{C}$ is called a left triangulated category if it is equipped with an endofunctor $\Omega: \mathscr{C} \rightarrow \mathscr{C}$ (not necessarily an auto-equivalence) and a class of diagrams $\Omega Z \rightarrow X \rightarrow Y \rightarrow Z$ called left triangle which satisfy the axioms of a triangulated category except that the left triangles may only be 'translated' to the left in (TR2). See [3, Definitions 2.2 and 2.3] for details. Right triangulated categories are defined similarly.

Lemma 3. Under the assumptions of Setup 2 we have:

(i) $\mathscr{A}$ is a left triangulated subcategory $\mathscr{T}$ which is closed under coproducts and extensions.

(ii) $\mathscr{B}$ is a right triangulated subcategory of $\mathscr{T}$ which is closed under products and extensions. 
Proof. This is immediate from Setup 2, note that in (i) the endofunctor is given by $\Omega=\Sigma^{-1}$ restricted to $\mathscr{A}$, and in (ii) the endofunctor is simply the suspension functor $\Sigma$ restricted to $\mathscr{B}$.

Let $\mathscr{F}$ be a full subcategory of a triangulated category $\mathscr{T}$. A morphism $\varphi: X \rightarrow F$ with $F \in \mathscr{F}$ is called a left $\mathscr{F}$-approximation if the induced morphism $\operatorname{Hom}_{\mathscr{T}}\left(\varphi, F^{\prime}\right): \operatorname{Hom}_{\mathscr{T}}\left(F, F^{\prime}\right) \rightarrow \operatorname{Hom}_{\mathscr{T}}\left(X, F^{\prime}\right)$ is surjective for all $F^{\prime} \in \mathscr{F}$. Dually, one obtains a right $\mathscr{F}$-approximation. Left $\mathscr{F}$-approximations are often called $\mathscr{F}$-preenvelopes and right $\mathscr{F}$-approximations are often called $\mathscr{F}$ precovers. The full subcategory $F$ is called contravariantly (resp., covariantly) finite if any object of $\mathscr{T}$ admits a right (resp., left) $\mathscr{F}$-approximation.

Lemma 4. $\operatorname{Add}(\mathscr{R})$ is contravariantly finite in $\mathscr{T}$ and for any $X \in \mathscr{T}$ there exists a distinguished triangle $R_{0} \stackrel{f_{0}}{\longrightarrow} X \stackrel{g_{0}}{\longrightarrow} B_{1} \stackrel{h_{0}}{\longrightarrow} \Sigma R_{0}$ in $\mathscr{T}$ such that

(i) $f_{0}$ is a right $\operatorname{Add}(\mathscr{R})$-approximation of $X$;

(ii) $0 \rightarrow \operatorname{Hom}_{\mathscr{T}}\left(\mathscr{R}, \Sigma^{n} B_{1}\right) \rightarrow \operatorname{Hom}_{\mathscr{T}}\left(\mathscr{R}, \Sigma^{n+1} R_{0}\right) \rightarrow \operatorname{Hom}_{\mathscr{T}}\left(\mathscr{R}, \Sigma^{n+1} X\right) \rightarrow 0$ is a short exact sequence for all $n \geqslant 0$.

(iii) The morphism $\operatorname{Hom}_{\mathscr{T}}\left(\Sigma^{-n} g_{0}, \mathscr{B}\right): \operatorname{Hom}_{\mathscr{T}}\left(\Sigma^{-n} B_{1}, \mathscr{B}\right) \rightarrow \operatorname{Hom}_{\mathscr{T}}\left(\Sigma^{-n} X, \mathscr{B}\right)$ is an isomorphism for all $n>0$ and a surjection for $n=0$.

Proof. One simply dualises the argument of [4, Lemma III.2.2].

Theorem 5. Let $\mathscr{T}$ be a triangulated category with set indexed coproducts and $\mathscr{S}$ a set of compact objects of $\mathscr{T}$ as in Setup $\mathbb{2}$. Then the pair of full subcategories defined in Setup 2,

$$
\begin{aligned}
\mathscr{A} & =\Sigma\left({ }^{\perp} \mathscr{B}\right) ; \\
\mathscr{B} & =\left\{X \in \mathscr{T} \mid \operatorname{Hom}_{\mathscr{T}}\left(S, \Sigma^{n} X\right)=0 \text { for all } S \in \mathscr{S} \text { and } n>0\right\} .
\end{aligned}
$$

defines a co-t-structure of finite type on $\mathscr{T}$.

Proof. We follow the proof of [4, Theorem III.2.3]. For an object $X \in \mathscr{T}$, by Lemma 4 we can inductively construct distinguished triangles

$$
R_{n} \stackrel{f_{n}}{\longrightarrow} B_{n} \stackrel{g_{n}}{\longrightarrow} B_{n+1} \stackrel{h_{n}}{\longrightarrow} \Sigma R_{n}
$$

for $n \geqslant 0$, where $B_{0}=X$. From these triangles, one obtains a tower of objects and morphisms,

$$
X=B_{0} \stackrel{g_{0}}{\longrightarrow} B_{1} \stackrel{g_{1}}{\longrightarrow} B_{2} \stackrel{g_{2}}{\longrightarrow} B_{3} \stackrel{g_{3}}{\longrightarrow} \cdots \longrightarrow B_{n} \stackrel{g_{n}}{\longrightarrow} B_{n+1} \longrightarrow \cdots .
$$

Recall from [14], for instance, that the homotopy colimit of the tower (4) is given by the distinguished triangle

$$
\coprod_{i=0}^{\infty} B_{i} \stackrel{1-\text { shift }}{\longrightarrow} \coprod_{i=0}^{\infty} B_{i} \longrightarrow \underset{\text { holim }}{\longrightarrow} B_{i} \longrightarrow \Sigma \coprod_{i=0}^{\infty} B_{i} .
$$


This induces a morphism $g_{X}: X \rightarrow \underline{\text { holim }} B_{n}$.

Note that in the proof of [4, Theorem III.2.3] it is shown that this is the reflection of $X$ along the inclusion functor $\iota: \mathscr{B} \rightarrow \mathscr{T}$, and hence one obtains a left adjoint and a $t$-structure. Here, this argument doesn't apply because Lemma 4 is not an exact dual of the corresponding lemma in [4]. However, it is sufficient to prove that the morphism $g_{X}: X \rightarrow \operatorname{holim} B_{n}$ is a left $\mathscr{B}$ approximation. First we must verify that $\stackrel{\operatorname{holim}}{\longrightarrow} B_{n}$ is indeed in $\mathscr{B}$.

By construction, the morphism

$$
\operatorname{Hom}_{\mathscr{T}}\left(\mathscr{R}, \Sigma^{i}\left(g_{n}\right)\right): \operatorname{Hom}_{\mathscr{T}}\left(\mathscr{R}, \Sigma^{i} B_{n-1}\right) \rightarrow \operatorname{Hom}_{\mathscr{T}}\left(\mathscr{R}, \Sigma^{i} B_{n}\right)
$$

is zero for all $n \geqslant 0$ and all $i>0$. It follows from the short exact sequence

$$
0 \rightarrow \coprod_{n \geqslant 0} \operatorname{Hom}_{\mathscr{T}}\left(\mathscr{R}, B_{n}\right) \rightarrow \coprod_{n \geqslant 0} \operatorname{Hom}_{\mathscr{T}}\left(\mathscr{R}, B_{n}\right) \rightarrow \underset{\lim }{\longrightarrow} \operatorname{Hom}_{\mathscr{T}}\left(\mathscr{R}, B_{n}\right) \rightarrow 0
$$

that $\lim _{\longrightarrow} \operatorname{Hom}_{\mathscr{T}}\left(\mathscr{R}, B_{n}\right)=0$. By [14, Lemma 2.8] we have an isomorphism $\lim _{\longrightarrow} \operatorname{Hom}_{\mathscr{T}}\left(\mathscr{R}, B_{n}\right) \cong \operatorname{Hom}_{\mathscr{T}}\left(\mathscr{R}, \underline{\text { holim}}_{\longrightarrow} B_{n}\right)$ since $\mathscr{R}$ consists of compect objects, hence we obtain $\operatorname{Hom}_{\mathscr{T}}\left(\mathscr{R}, \underline{\text { holim }} B_{n}\right)=0$ and holim $B_{n} \in \mathscr{B}$.

Now we prove that $g_{X}: X \rightarrow$ holim $B_{n}$ is a left $\overrightarrow{\mathscr{B}}$-approximation by following the proof of [15, Proposition 4.2]. Let $B^{\prime} \in \mathscr{B}$ and consider the distinguished triangle (3). By Lemma 4, the morphism

$$
\operatorname{Hom}_{\mathscr{T}}\left(\Sigma^{i}\left(g_{n}\right), B^{\prime}\right): \operatorname{Hom}_{\mathscr{T}}\left(\Sigma^{i} B_{n}, B^{\prime}\right) \rightarrow \operatorname{Hom}_{\mathscr{T}}\left(\Sigma^{i} B_{n-1}, B^{\prime}\right)
$$

arising from (3) is an isomorphism for $i<0$ and a surjection for $i=0$. In particular, given a map $\beta_{0}: X \rightarrow B^{\prime}$, the fact that we have a surjection for $i=0$ yields the following commutative diagram:

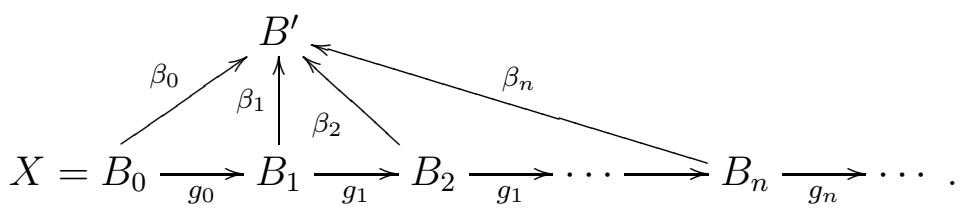

By construction, the composite

$$
\coprod_{i=0}^{\infty} B_{i} \stackrel{1-\text { shift }}{\longrightarrow} \coprod_{i=0}^{\infty} B_{i} \stackrel{\left\langle\beta_{i}\right\rangle}{\longrightarrow} B^{\prime}
$$

is zero, where $\left\langle\beta_{i}\right\rangle$ is the unique map arising from the coproduct. Thus, we obtain the following commutative diagram

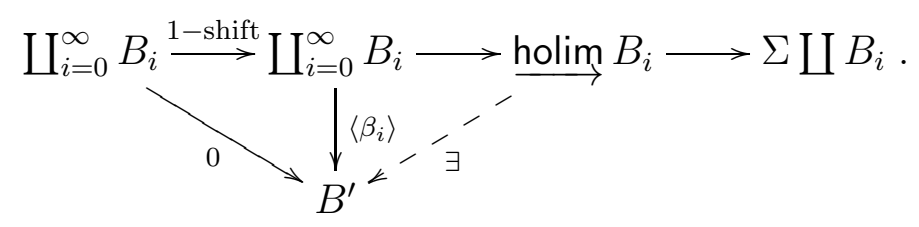


It follows that $g_{X}: X \rightarrow \underline{\text { holim }} B_{n}$ is a left $\mathscr{B}$-approximation, as claimed.

Now we need to verify that $(\mathscr{A}, \mathscr{B})$ as defined in Setup 2 is a co-t-structure on $\mathscr{T}$. Conditions $(0),(1)$ and (2) are clear. In order to prove (3) we need the following lemma.

Lemma 6. The morphism $\operatorname{Hom}_{\mathscr{T}}\left(\Sigma^{i} g_{X}, \mathscr{B}\right): \operatorname{Hom}_{\mathscr{T}}\left(\Sigma^{i} B, \mathscr{B}\right) \rightarrow \operatorname{Hom}_{\mathscr{T}}\left(\Sigma^{i} X, \mathscr{B}\right)$, where $B=$ holim $B_{n}$, is an isomorphism for $i<0$.

Proof of lemma. Apply the functor $\operatorname{Hom}_{\mathscr{T}}\left(\Sigma^{i}-, \mathscr{B}\right)$ for $i<0$ to the tower (4) above to get the inverse tower

$$
\cdots \rightarrow \operatorname{Hom}_{\mathscr{T}}\left(\Sigma^{i} B_{2}, \mathscr{B}\right) \rightarrow \operatorname{Hom}_{\mathscr{T}}\left(\Sigma^{i} B_{1}, \mathscr{B}\right) \rightarrow \operatorname{Hom}_{\mathscr{T}}\left(\Sigma^{i} B_{0}, \mathscr{B}\right)
$$

By Lemma 4, each morphism in the tower (5) is an isomorphism, so we have $\lim _{\operatorname{Hom}}\left(\Sigma^{i}, \mathscr{B}\right) \cong \operatorname{Hom}_{\mathscr{T}}\left(\Sigma^{i} B_{0}, \mathscr{B}\right)$ for $i<0$. By [2, Lemma 5.8], there is a short exact sequence

$$
\lim ^{1} \operatorname{Hom}_{\mathscr{T}}\left(\Sigma^{i+1} B_{n}, \mathscr{B}\right) \hookrightarrow \operatorname{Hom}_{\mathscr{T}}\left(\Sigma^{i} \underline{\text { holim}}_{\longrightarrow} B_{n}, \mathscr{B}\right) \rightarrow \underset{\lim }{\longleftarrow} \operatorname{Hom}_{\mathscr{T}}\left(\Sigma^{i} B_{n}, \mathscr{B}\right)
$$

for $i<0$, where $\lim ^{1}$ denotes the first right derived functor of $\lim$; see [17]. For $i \leqslant 0$, the tower (5) consists of surjective morphisms (for $i<0$, isomorphisms), it particular, it satisfies the Mittag-Leffler condition. It follows by [17] that $\lim ^{1} \operatorname{Hom}_{\mathscr{T}}\left(\Sigma^{i+1} B_{n}, \mathscr{B}\right)=0$, so that

$$
\operatorname{Hom}_{\mathscr{T}}\left(\Sigma^{i} \underline{\text { holim }} B_{n}, \mathscr{B}\right) \stackrel{\sim}{\longrightarrow} \lim \operatorname{Hom}_{\mathscr{T}}\left(\Sigma^{i} B_{n}, \mathscr{B}\right)
$$

gives the desired isomorphism.

Now we return to the proof of Theorem 5 and the verification of condition (3) in definition of a co- $t$-structure. Let $X \in \mathscr{T}$ and take the left $\mathscr{B}$-approximation $g_{X}: X \rightarrow B$, where $B=\underline{\text { holim }} B_{n}$. Extend this morphism to a distinguished triangle:

$$
\Sigma^{-1} A \rightarrow X \rightarrow B \rightarrow A .
$$

Applying the functor $\operatorname{Hom}_{\mathscr{T}}(-, \mathscr{B})$ to (6) and using Lemma 6, one can read off from the resulting long exact sequence that $\operatorname{Hom}_{\mathscr{T}}\left(\Sigma^{-1} A, \mathscr{B}\right)=0$, i.e. $A \in \mathscr{A}$, as desired.

Since $\mathscr{S}$ is consists of compact objects, then $\mathscr{B}$ is closed under set indexed coproducts, and hence $(\mathscr{A}, \mathscr{B})$ is a co-t-structure of finite type on $\mathscr{T}$.

In [7, Definition 4.4.1] a co-t-structure $(\mathscr{A}, \mathscr{B})$ is called left adjacent to a (co)$t$-structure $(\mathscr{X}, \mathscr{Y})$ if $\mathscr{B}=\mathscr{X}$; cf. the notion of 'torsion torsion-free triple' in [4]. The notion of right adjacency is defined analogously.

Corollary 7. Under the additional hypotheses

(1) $\operatorname{Hom}_{\mathscr{T}}\left(S, \Sigma^{i} S^{\prime}\right)=0$ for all $S, S^{\prime} \in \mathscr{S}$ and for all $i>0$; 
(2) $\left\{\Sigma^{i} S \mid i \in \mathbb{Z}, S \in \mathscr{S}\right\}$ is a generating set;

then the co-t-structure of Theorem 5 is left adjacent to the t-structure obtained in [4, Proposition III.2.8] (cf. (11) and Proposition 12).

Example 8. Consider the homotopy category of spectra $\mathrm{Ho}(\mathrm{Sp})$ and let $\mathscr{S}=$ $\left\{S^{0}\right\}$ consist of only the sphere spectrum. Then $S^{0}$ is compact and satisfies the hypotheses of [4, Proposition III.2.8] (see [12], for instance). Thus it follows that the co- $t$-structure induced by Theorem 5 is left adjacent to the natural $t$-structure on $\mathrm{Ho}(\mathrm{Sp})$.

Remark 9. Theorem 5 is established for arbitrary sets of objects $\mathscr{S}$ (whether compact or not) in the case that $\mathscr{T}$ is an 'efficient' algebraic triangulated category; see Corollary 3.5 and Proposition 3.15 of [16].

We next obtain an analogue of [4, Proposition III.2.8]. We add the following conditions to Setup 2:

Setup 10. Suppose in addition to the conditions satisfied in Setup 2 the set of objects $\mathscr{S}$ also satisfies:

(1) $\operatorname{Hom}_{\mathscr{T}}\left(\Sigma^{i} S, S^{\prime}\right)=0$ for $i>0$ and all objects $S$ and $S^{\prime}$ in $\mathscr{S}$;

(2) $\operatorname{Hom}_{\mathscr{T}}\left(S, \Sigma S^{\prime}\right)=0$ for all objects $S$ and $S^{\prime}$ in $\mathscr{S}$.

The construction here is slightly different from that of Theorem 5 and hinges on the following technical lemma.

Lemma 11. Under the conditions of Setups 2 and 10, for any object $X$ in $\mathscr{T}$ there is a left $\mathscr{B}$-approximation $\beta: X \rightarrow B$ with $B \in \mathscr{B}=\mathscr{R}^{\perp}$ such that $\operatorname{Hom}_{\mathscr{T}}\left(S, \Sigma^{i}(\beta)\right): \operatorname{Hom}_{\mathscr{T}}\left(S, \Sigma^{i} X\right) \rightarrow \operatorname{Hom}_{\mathscr{T}}\left(S, \Sigma^{i} B\right)$ is an isomorphism for $i<1$ and all $S \in \mathscr{S}$.

Proof. Note that in [15, Proposition 4.1] the hypothesis that $\operatorname{End}(S)$ is a division ring (for all $S \in \mathscr{S}$ ) is not required, and so the lemma follows by [15, Proposition 4.2 and Lemma 5.1].

Proposition 12. Under the conditions of Setups 2 and 10 we have

$$
\mathscr{A} \subseteq\left\{X \in \mathscr{T} \mid \operatorname{Hom}_{\mathscr{T}}\left(S, \Sigma^{i} X\right)=0 \text { for } i<0, S \in \mathscr{S}\right\} .
$$

Moreover, the following statements are equivalent:

(i) The set $\left\{\Sigma^{i} S \mid i \in \mathbb{Z}, S \in \mathscr{S}\right\}$ is a generating set for $\mathscr{T}$.

(ii) $\mathscr{A}=\left\{X \in \mathscr{T} \mid \operatorname{Hom}_{\mathscr{T}}\left(S, \Sigma^{i} X\right)=0\right.$ for $\left.i<0, S \in \mathscr{S}\right\}$.

Proof. Suppose $(\mathscr{A}, \mathscr{B})$ is the co-t-structure on $\mathscr{T}$ induced in Theorem 5, Let $\overline{\mathscr{A}}=\left\{X \in \mathscr{T} \mid \operatorname{Hom}_{\mathscr{T}}\left(S, \Sigma^{i} X\right)=0\right.$ for $\left.i<0, S \in \mathscr{S}\right\}$; in order to show that $\mathscr{A} \subseteq \mathscr{A}$, it is sufficient to show that

$$
\Sigma^{-1} \mathscr{A} \subseteq \Sigma^{-1} \overline{\mathscr{A}}=\left\{X \in \mathscr{T} \mid \operatorname{Hom}_{\mathscr{T}}\left(S, \Sigma^{i} X\right)=0 \text { for } i<1, S \in \mathscr{S}\right\}
$$


Let $X \in \Sigma^{-1} \mathscr{A}$ and consider the left $\mathscr{B}$-approximation $\beta: X \rightarrow B$ arising from Lemma 11. By definition, the induced map $\operatorname{Hom}_{\mathscr{T}}\left(\beta, B^{\prime}\right): \operatorname{Hom}_{\mathscr{T}}\left(B, B^{\prime}\right) \rightarrow$ $\operatorname{Hom}_{\mathscr{T}}\left(X, B^{\prime}\right)$ is a surjection for all $B^{\prime} \in \mathscr{B}$. Since $X \in \Sigma^{-1} \mathscr{A}={ }^{\perp} \mathscr{B}$, we have $\operatorname{Hom}_{\mathscr{T}}\left(X, B^{\prime}\right)=0$ for all $B^{\prime} \in \mathscr{B}$. In particular, setting $B^{\prime}=B$ yields $\beta=0$. Now by Lemma 11, we have the following isomorphism:

$$
\operatorname{Hom}_{\mathscr{T}}\left(S, \Sigma^{i}(\beta)\right): \operatorname{Hom}_{\mathscr{T}}\left(S, \Sigma^{i} X\right) \stackrel{\sim}{\longrightarrow} \operatorname{Hom}_{\mathscr{T}}\left(S, \Sigma^{i} B\right)
$$

for all $S \in \mathscr{S}$ and $i<1$. Since $\beta=0$, it follows that the induced isomorphism $\operatorname{Hom}_{\mathscr{T}}\left(S, \Sigma^{i}(\beta)\right)=0$ for all $i<1$, in which case we must have $\operatorname{Hom}_{\mathscr{T}}\left(S, \Sigma^{i} X\right)=$ 0 for all $S \in \mathscr{S}$ and $i<1$, i.e. $X \in \Sigma^{-1} \overline{\mathscr{A}}$, giving the desired inclusion.

$(i) \Longrightarrow(i i)$. Suppose that $\left\{\Sigma^{i} S \mid i \in \mathbb{Z}, S \in \mathscr{S}\right\}$ is a generating set for $\mathscr{T}$. We claim that $\mathscr{A}=\mathscr{A}$, we have shown the inclusion $\mathscr{A} \subseteq \mathscr{A}$ above so we only need to verify that $\overline{\mathscr{A}} \subseteq \mathscr{A}$. Let $X \in \mathscr{A}$; by Lemma 11 , there is a left $\mathscr{B}$-approximation $\beta: \Sigma^{-1} X \rightarrow B$ with $B \in \mathscr{B}$, namely, for any $B^{\prime} \in \mathscr{B}$ we have a surjection $\operatorname{Hom}_{\mathscr{T}}\left(B, B^{\prime}\right) \rightarrow \operatorname{Hom}_{\mathscr{T}}\left(\Sigma^{-1} X, B^{\prime}\right)$. We use the argument of [15. Theorem 5.1], namely, we have the following isomorphism and equalities:

$$
\begin{aligned}
\operatorname{Hom}_{\mathscr{T}}\left(S, \Sigma^{i-1} X\right) & \cong \operatorname{Hom}_{\mathscr{T}}\left(S, \Sigma^{i} B\right) \text { for all } i<1 \text { and } S \in \mathscr{S} \text { (Lemma 11) } \\
\operatorname{Hom}_{\mathscr{T}}\left(S, \Sigma^{i-1} X\right) & =0 \text { for all } i<1 \text { and } S \in \mathscr{S}(\text { since } X \in \mathscr{A}) \\
\operatorname{Hom}_{\mathscr{T}}\left(S, \Sigma^{i} B\right) & =0 \text { for all } i>0 \text { and } S \in \mathscr{S}(\text { since } B \in \mathscr{B}) .
\end{aligned}
$$

It follows that $\operatorname{Hom}_{\mathscr{T}}\left(S, \Sigma^{i} B\right)=0$ for all $i \in \mathbb{Z}$, and thus, since $\left\{\Sigma^{i} S \mid i \in \mathbb{Z}, S \in\right.$ $\mathscr{S}\}$ is a generating set in $\mathscr{T}$, we have $B=0$. Hence $\operatorname{Hom}_{\mathscr{T}}\left(B, B^{\prime}\right)=0$ for all $B^{\prime} \in \mathscr{B}$, in which case, we have $\operatorname{Hom}_{\mathscr{T}}\left(\Sigma^{-1} X, B^{\prime}\right)=0$. Thus $\Sigma^{-1} X \in{ }^{\perp} \mathscr{B}$, i.e. $X \in \mathscr{A}$.

$($ ii $) \Longrightarrow(i)$. Let $X$ be an object of $\mathscr{T}$ such that $\operatorname{Hom}_{\mathscr{T}}\left(\Sigma^{i} S, X\right)=0$ for all $i \in \mathbb{Z}$ and $S \in \mathscr{S}$. Then $X \in \mathscr{B}$ and $X \in \Sigma^{-1} \mathscr{A}$, i.e. $X \in \Sigma^{-1} \mathscr{A} \cap \mathscr{B}=\{0\}$, whence $\left\{\Sigma^{i} S \mid i \in \mathbb{Z}, S \in \mathscr{S}\right\}$ is a generating set for $\mathscr{T}$.

Remark 13. (1) Note that the argument of [4] does not apply here because one also requires the vanishing of $\operatorname{Hom}_{\mathscr{T}}\left(S, \Sigma S^{\prime}\right)$ for all $S$ and $S^{\prime}$ in $\mathscr{S}$. To ensure this following the argument of [4] would require assuming that $\operatorname{End}(S)=0$. The condition $\operatorname{Hom}_{\mathscr{T}}\left(S, \Sigma S^{\prime}\right)$ for all $S$ and $S^{\prime}$ in $\mathscr{S}$ is used in the construction of the left $\mathscr{B}$-approximation in Lemma 11 .

(2) Under the hypotheses of Setups 2 and 10 , Proposition 12 gives the existence of a co-t-structure right adjacent to that obtained in [4, Theorem III.2.3], cf. (2).

In the following example we look at the canonical co- $t$-structure induced by a connected cochain differential graded algebra (DGA); for details regarding DGAs see [5] and [8], also see the motivation presented in [15]. In particular, note that such DGAs arise naturally as the cochain algebras of (simply) connected CW-complexes; see [8]. 
Example 14. Let $R$ be a connected cochain DGA, i.e $H^{i}(R)=0$ for $i<0$ and $H^{1}(R)=0$, and let $\mathscr{D}(R)$ denote its (unbounded) derived category of differential graded (DG) left $R$-modules. Observe that $H^{i}(M) \cong \operatorname{Hom}_{\mathscr{D}(R)}\left(R, \Sigma^{i} M\right)$ for all $i \in \mathbb{Z}$, thus $R$ clearly satisfies the conditions of Proposition $[12$, and as such one obtains a co-t-structure on $\mathscr{D}(R)$.

Acknowledgement. The author would like to thank María José Souto Salorio for correcting an error in Corollary 7.

\section{REFERENCES}

[1] P. N. Achar and D. Treumann, Purity and decomposition theorems for staggered sheaves; preprint, available at arXiv:0808.3210v1, 23 Aug 2008.

[2] A. Beligiannis, Relative Homological Algebra and Purity in Triangulated Categories; J. Algebra 227 (2000), 268-361.

[3] A. Beligiannis and N. Marmardis, Left triangulated categories arising from contravariantly finite subcategories; Comm. Algebra 22 (12) (1994), 5021-5036.

[4] A. Beligiannis and I. Reiten, Homological and Homotopical Aspects of Torsion Theories; Memoirs of the American Mathematical Society, Vol. 188 (2007).

[5] J. Bernstein and V. Lunts, "Equivariant Sheaves and Functors"; Lecture Notes in Mathematics 1578, Springer-Verlag, Berlin Heidelberg, 1994.

[6] M. V. Bondarko, Motivically functorial Coniveau spectral sequences; direct summands of cohomology of function fields; Doc. Math., Vol. Extra Volume: Andrei A. Suslin's Sixtieth Birthday (2010), 33-117.

[7] M. V. Bondarko, Weight structures vs. t-structures: weight filtrations, spectral sequences and complexes (for motives and in general); to appear in J. K-theory, preprint available at arXiv:0704.4003v7, 3 Jul 2010.

[8] Y. Félix, S. Halperin and J-C. Thomas, "Rational Homotopy Theory"; Graduate Texts in Mathematics 205, Springer, New York, 2001.

[9] T. Holm and P. Jørgensen, Triangulated categories: definitions, properties and examples; in "Triangulated Categories", London Mathematical Society Lecture Note Series 375, CUP, Cambridge, 2010.

[10] M. Hoshino, Y. Kato and J. Miyachi, On t-structures and torsion theories induced by compact objects; J. Pure Appl. Algebra 167 (2002), 15-35.

[11] O. Iyama and Y. Yoshino, Mutation in triangulated categories and rigid Cohen-Macaulay modules; Invent. Math. 172 (2008) no. 1, 117-168.

[12] H. R. Margolis, "Spectra and the Steenrod Algebra"; North-Holland Mathematical Library, 29, North-Holland Publishing Co., Amsterdam, 1983.

[13] O. Mendoza, E. C. Sáenz, V. Santiago and M. J. Souto Salorio, Auslander-Buchweitz Context and Co-t-structures; preprint, available at arXiv:1002.4604v1, 24 Feb 2010.

[14] A. Neeman, The Grothendieck duality theorem via Bousfield's techniques and Brown representability; J. Amer. Math. Soc. 9 (1996), 205-236.

[15] D. Pauksztello, Compact corigid objects in triangulated categories and co-t-structures; Cent. Eur. J. Math. 6 (2008), 25-42.

[16] M. Saorín and J. Šťovíček, On exact categories and applications to triangulated adjoints and model structures; preprint, available at: arXiv:1005.3248v1, 18 May 2010. 
[17] C. A. Weibel, "An Introduction to Homological Algebra"; Cambridge studies in advanced mathematics 38, CUP, Cambridge, 1994.

[18] J. Wildeshaus, Chow motives without projectivity; Compositio Math. 145 (2009), 11961226 .

[19] J. Wildeshaus, Notes on Artin-Tate motives; preprint, available at: arXiv:0811.4551v2, 14 Jan 2010.

Institut FÜr Algebra, Zahlentheorie und Diskrete Mathematik, Fakultät Für Mathematik und Physik, Leibniz Universität Hannover, Welfengarten 1, 30167 Hannover, Germany.

E-mail address: pauk@math.uni-hannover.de 